\title{
A HIGH PERFORMANCE 8 GHZ, 8 PSK DIGITAL RADIO
}

\author{
Giuseppe G. Russo \\ Paul R. Hartmann \\ Collins Transmission Systems Division \\ Rockwell International \\ Richardson, Texas, U.S.A.
}

\begin{abstract}
Rapid growth and modernization of microwave communications is currently taking place in both the commercial and military environments. Much of this growth is being accomplished through digital transmission, and calls for efficient utilization of frequency spectra allocated for LOS communications. As the bit efficiencies and data rates of digital radio systems have increased, the problems associated with multipath propagation phenomena have become more evident. This is due to the fact that the transmitter signal is randomized before transmission and the system behaves as though it has full loading at all times. To minimize outages and to meet path availability objectives, diversity protection alone, in many instances, is not sufficient. However, diversity protection, coupled with an acaptive equalizer, will meet the objective in all but a few severe cases.
\end{abstract}

This paper describes a digital radio system operating in the $8 \mathrm{GHz}$ band with 8 PSK modulation and nominal data rates of $45 \mathrm{Mb} / \mathrm{s}$ or $90 \mathrm{Mb} / \mathrm{s}$. Multipath propagation and its effects on digital transmission are discussed. Improvements in bit error rate threshold obtained through the use of an adaptive equalizer designed into the receiver are also presented.

\section{INTRODUCTION}

The recognized benefits of digital transmission include: transmission quality, ease of encription, reduced system cost, and direct interface with digital switching systems. These factors constitute some of the driving forces that account for the rapid expansion of digital transmission. This modern, high-performance digital microwave radio, MDR-8-( ), uses 8 PSK digital modulation at RF, and can be configured to transmit one or two $44.736 \mathrm{Mb} / \mathrm{s}$ DS-3 signals in a $20 \mathrm{MHz}$ or $40 \mathrm{MHz}$ RF bandwidth on a single polarization. These configurations correspond to 672 and 1344 PCM voice channels, respectively. When auxiliary channels and overhead bits are multiplexed with the DS-3 data stream, the spectrum efficiency is $2.26 \mathrm{bits} / \mathrm{Hz}$. 
Frequency diversity or hot stand-by protection configurations are easily implemented, due to the straightforward RF architecture. To compensate for distortion effects due to multipath propagation, the receiver uses an IF amplifier containing a recently-developed adaptive equalizer.

\section{SYSTEM DESCRIPTION}

The MDR-8-( ) microwave digital radio operates in the $7.125-8.500 \mathrm{GHz}$ band, and can be configured for frequency diversity or hot standby protection. The system is all solid state, except for the high efficiency TWT power amplifiers. The general characteristics are shown in table 1. The MDR-8-( ) rack structure, shown in figure 1, contains two transmitters, two receivers, the DS-3 interface, the order-wire, and protection equipment. The mechanical configuration of the MDR-8-( ) features front access for all modules, card cages, waveguide, and rack wiring, and removable front covers. The following description is of the MDR-8-5N narrow-band system; the wide-band system is an easy extension of the one described. [1]

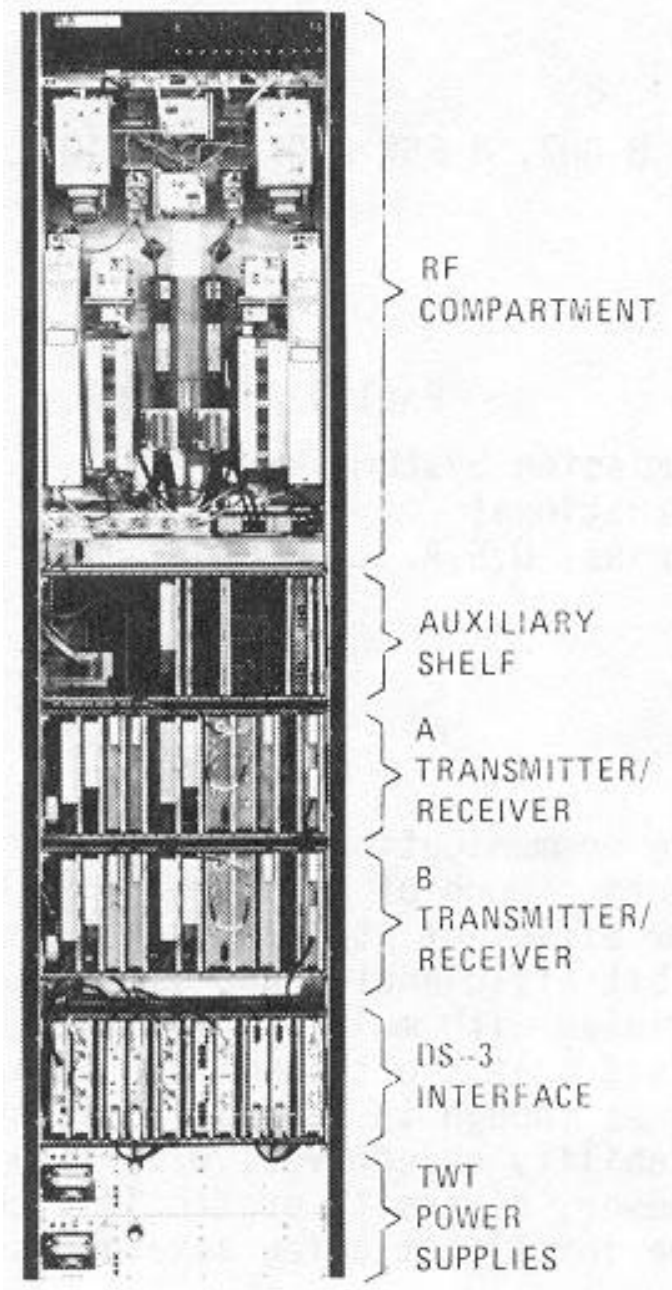

Figure 1.

MDR-8 SUBSYSTEM IDENTIFICATION (FREQUENCY DIVERSITY SHOWN) 


\section{DS-3 INTERFACE}

As shown in figure 2, the bipolar signal at the $44.736 \mathrm{Mb} / \mathrm{s}$ data rate is applied to the DS-3 interface unit. The primary function of the interface is that of conditioning the DS-3 input signal to the required MDR-8-5N system format. This unit is used only at terminals and at repeaters with drops. The transmitter portion converts the bipolar signal into an NRZ signal, removes the bipolar three zero substitution (B3ZS) encoding, and recovers the clock. Bit stuffing techniques are used for the system framing code and auxiliary channel bit locations. Three data lines plus the clock signal constitute the output of the DS-3 interface which is then applied to the RF transmitter. The receiver portion of the DS-3 interface receives the three data lines and the recovered system clock. The data lines are then combined and the overhead bits are removed (destuffing). The B3ZS coding and bipolar format are restored to meet the DSX-3 interface specification at $44.736 \mathrm{Mb} / \mathrm{s}$.

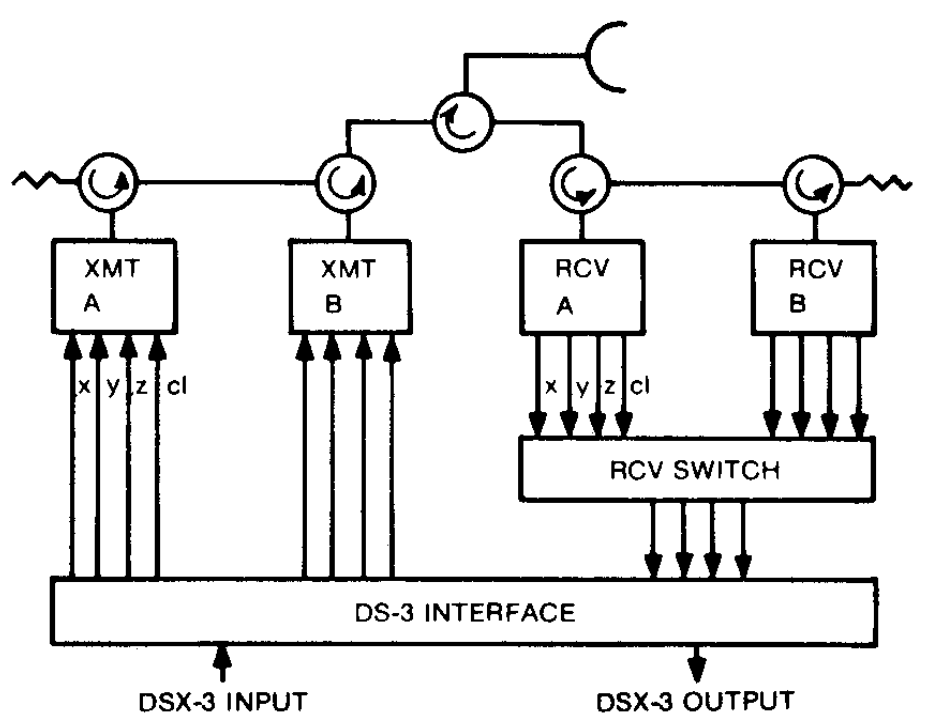

Figure 2. MDR-8-5N DIGITAL RADIO SYSTEM

\section{MDR-8-5( ) TRANSMITTER}

The MDR-8-5( ) transmitter is similar to a conventional remodulating microwave radio. The input signal to the transmitter comes from the DS-3 interface for a terminal or repeater with drops, and from the receivers decoder for a repeater without drops.

The block diagram of the MDR-8-5( ) transmitter is shown in figure 3. The auxiliary channel insert module inserts fault alarm and service channel information into the data streams, and provides protection for the radio frame. The encoder accepts the three data lines and clock from the auxiliary modulator. To prevent generation of line spectra, the data is scrambled and then differentially encoded. The output of the encoder is applied to the 8 PSK modulator. The modulator is a path length modulator, where the output of the 
RF source is phase modulated by the encoded data. The output of the modulator is then amplified in the high efficiency traveling wave tube amplifier. The amplified signal is then applied to the transmit filter for spectrum shaping. The longterm stability of the transmitter spectrum shape is achieved by using a sealed 5-element cylindrical cavity filter made of Invar. The transmitted RF spectrum is shown in figure 4, together with the Federal Communications Commission spectrum limits for a $20 \mathrm{MHz}$ wide RF channel.

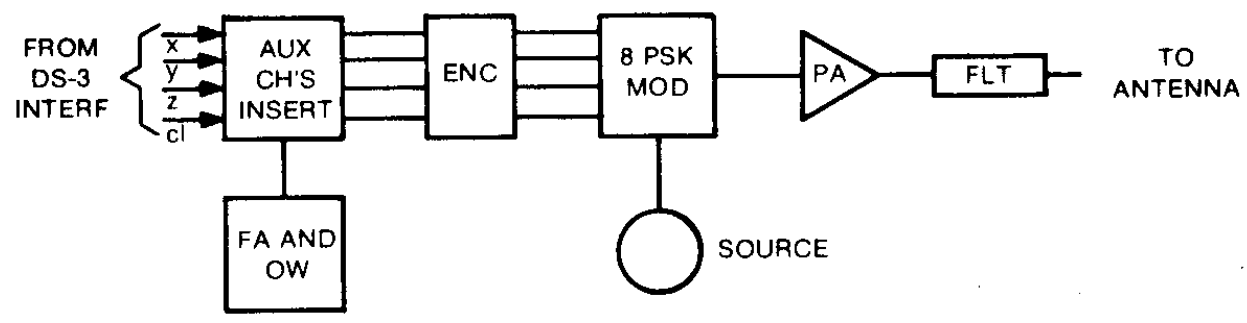

Figure 3.

MDR-8-5( ) TRANSMIT SUBSYSTEM

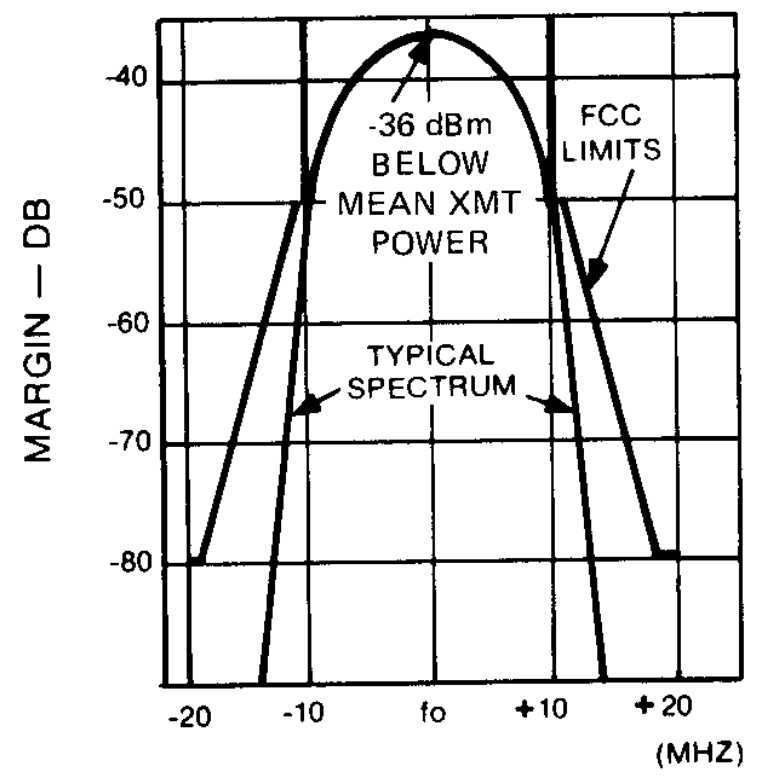

Figure 4.

MDR-8-5N SPECTRUM OCCUPANCY

\section{MDR-8-5( ) RECEIVER}

The digital receiver is a conventional superheterodyne receiver with a low-loss, sixelement cylindrical cavity preselector filter and low-noise single balanced hybrid mixer to achieve a low overall noise figure. A block diagram of the receiver is shown in figure 5.

The RF input signal is coupled through the preselector filter into the down converter mixer. The down converter module also contains an AGC amplifier to extend the dynamic range of the receiver. The $70 \mathrm{MHz}$ output of the mixer is then coupled to the IF amplifier. 


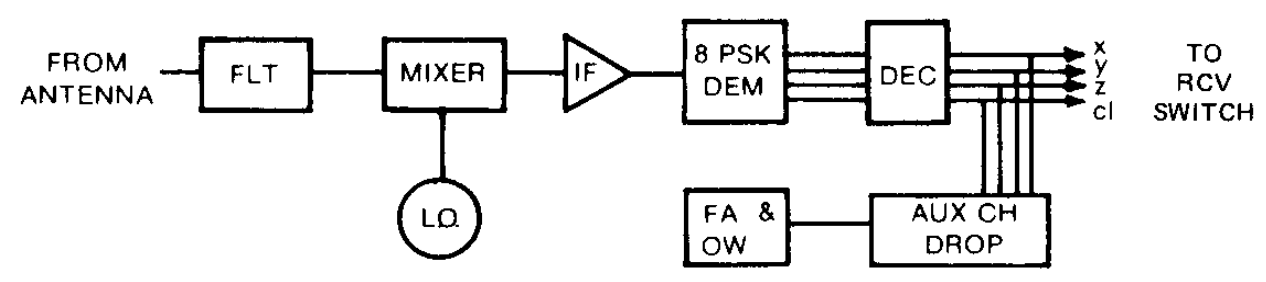

Figure 5. MDR-8-5N RECEIVER SUBSYSTEM

The $70 \mathrm{MHz}$ IF amplifier provides AGC amplification, system delay equalization, and establishes the receiver noise bandwidth. The AGC amplifier provides $55 \mathrm{~dB}$ of receiver dynamic range with a constant output level. In addition, the IF amplifier has an adaptive amplitude equalization capability that can automatically correct for linear slope and inband null resulting from multipath propagation.

Clock recovery, carrier recovery, and data detection are accomplished in the 8 PSK demodulator, together with prediction of RF channel performance obtained through an eye pattern monitoring circuit. Decoding and descrambling of the data streams occurs in the decoder unit to restore the three data lines applied to the digital transmitter input. The auxiliary channel drop module locates and extracts the twelve $32 \mathrm{~kb} / \mathrm{s}$ auxiliary channels and makes them available to the service channel and fault alarm equipment.

\section{SYSTEM PROTECTION}

The 1:1 switch and logic for the MDR-8-( ) is integrated into the radio bay. The switching logic provides 12 levels of receiver priority and 8 levels of transmitter priority. It provides error-free transfer of the receiver output signal for diversity and maintenance. The system is configured so that conversion to $1: \mathrm{N}$ can be accomplished with minimum changes.

\section{MULTIPATH PROPAGATION AND EFFECTS ON DIGITAL RADIO}

Microwave multipath propagation is a metereological phenomenon that has been recognized as a source of fading for many years. It arises from destructive interference between the primary signal ray and one or more reflected rays that arrive at the receiver antenna delayed in time from the primary signal. The path geometry for refraction due to a single layer is shown in figure 6 . Although the multipath propagation is a complex phenomenon, the use of a two-ray propagation mod 1 for laboratory studies has been proven to be a fair representation of this phenomenon. [2,3] During multipath conditions, deep fading arises from the fact that, at some frequency, the primary and reflected rays arrive out of phase. Amplitude and delay patterns for two-ray interference are shown in figure 7. Deep amplitude nulls occurring in the proximity of, or within the RF channel constitute the greatest problem. 

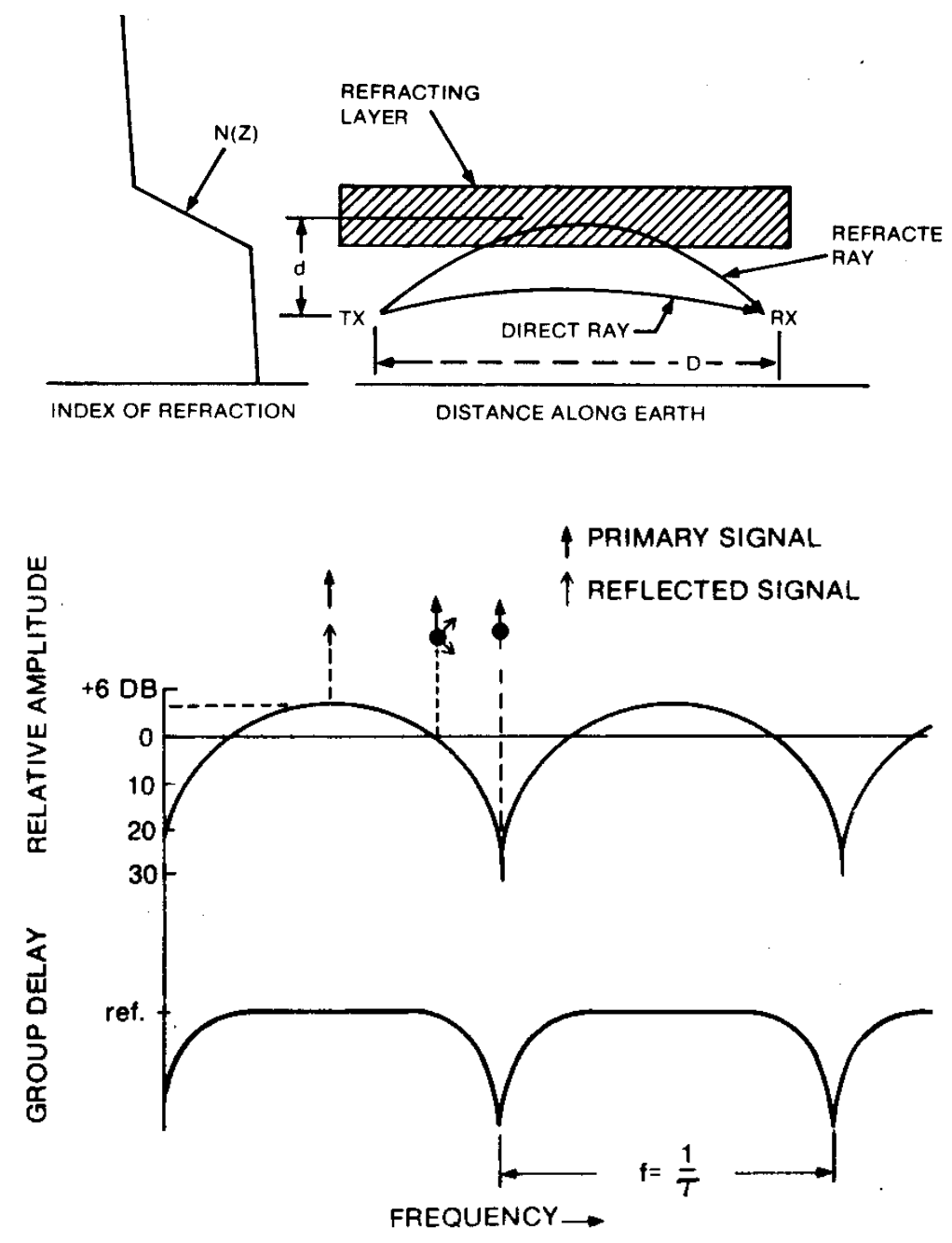

Figure 6.

PATH GEOMETRY FOR REFRACTION FROM A SINGLE LAYER
Figure 7.

AMPLITUDE AND DELAY CHARACTERISTICS IN MULTIPATH PROPAGATION

The degradation of BER on a digital system during multipath depends on the magnitude of the resulting passband distortion which, in turn, depends on the fade depth and the time delay between multipath signals. This degradation is caused by intersymbol interference resulting from frequency-dependent gain and group delay. Laboratory studies have shown that, for a given echo delay and fade depth, gain slope causes more severe BER threshold degradation than delay slope or parabolic delay. [4]

The adaptive equalizers used in the MDR-8-( ) radio system are quite effective in controlling multipath, since they compensate directly in the receiver for passband distortion created by multipath. [5] This compensation is made possible due to the broad and well-defined pseudorandom digital spectrum transmitted after scrambling the data.

\section{BER IMPROVEMENTS USING ADAPTIVE EQUALIZERS}

System BER threshold improvement using adaptive equalizers with multipath simulated by a two-ray model is shown in figure 8, where the effects of amplitude slope and in-band null are shown separately. The results show that, under multipath conditions creating amplitude 
slope or amplitude null, the system with the adaptive equalizer off, for most fades, is inoperative but, when the adaptive equalizer is turned on, the BER threshold performances under the same conditions are brought well within the BER specifications of the radio with no multipath.

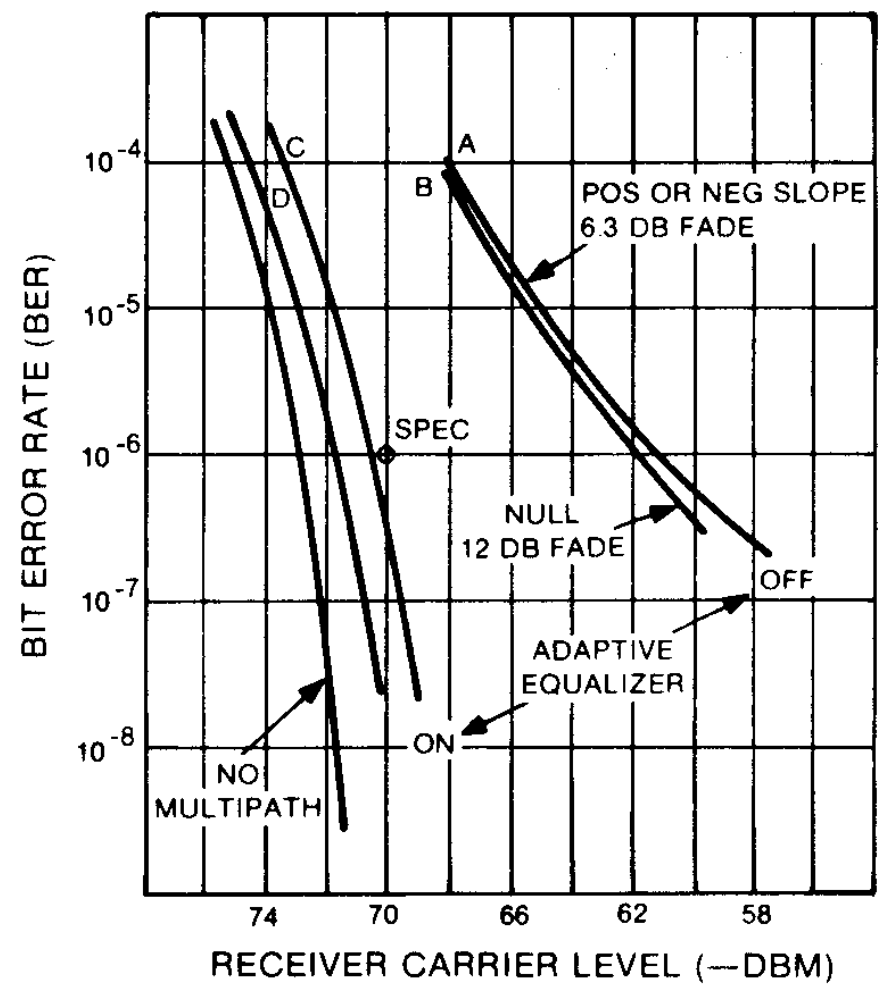

Figure 8.

BER IMPROVEMENTS USING ADAPTIVE EQUALIZER FOR LINEAR AMPLITUDE SLOPE $(A, C)$ AND FOR SYMMETRICAL NULL (B,D). MULTIPATH DELAY $=6.3$ NSEC.

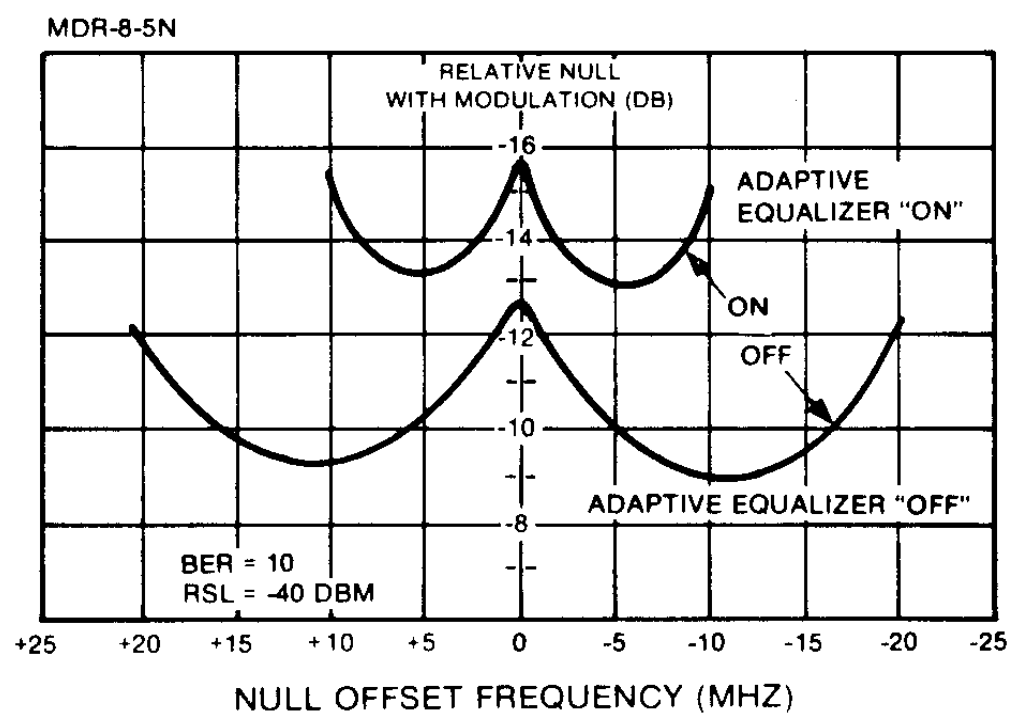

Figure 9.

SYSTEM SENSITIVITY TO MULTIPATH

PROPAGATION AT CONSTANT BER

The critical notch depth vs. offset frequency for a constant BER is shown in figure 9. This "W" shaped curve shown the sensitivity of the system when a multipath disturbance (null) sweeps across the bandpass. The improvement ( decrease in sensitivity to multipath) is 
measured by the offset frequency required and by the additional fade necessary to achieve the same level of BER measured without the adaptive equalizer. [6]

The adaptive equalizer used in the MDR-8-( ) is incorporated into the, $70 \mathrm{MHz}$ IF amplifier. It consists basically of two slope monitoring circuits tuned near the edges of the passband and a null detector tuned to the center of the passband. Utilization of these circuits reshapes the passband spectrum close to the non-multipath response.

\section{CONCLUSION}

The MDR-8-( ) microwave radio system just described is intended for use in short and medium haul applications, and is a high-performance, high-capacity system. The use of adaptive equalizers to counteract the detrimental effects of in-band distortion due to multipath propagation will improve the systems availability and performance margin.

\section{Table 1. MDR-8-5N SYSTEM CHARACTERISTICS}

Frequency Range

Transmit Stability

Modulation

Voice Circuits

Data Input/Output

System Data Rate

Receive Noise Figure

Receiver Level

Receiver Threshold

(for $10^{-6}$ ) BER)

Power Output ${ }^{(2)}$

Reduced

Normal

System Gain

(@ $\left.\mathrm{BER}=10^{-6}\right)$
$7.125-8.500 \mathrm{GHz}$

$\pm 0.0005 \%$

8 PSK

$672^{(1)}$

One DSX-3

45.129 Mb/s Nominal

$8.8 \mathrm{~dB}$ Maximum ${ }^{(2)}$

$-8 \mathrm{dBm}$ Maximum

$-70 \mathrm{dBm}$ Maximum ${ }^{(2)}$

(1) North American Standard

(2) At Branching Circulator

Frequency Hot

Diversity $\quad \underline{\text { Standby }}$

$+30 \mathrm{dBm} \quad+33 \mathrm{dBm}$ min.

$+35.5 \mathrm{dBm} \quad+38.5 \mathrm{dBm}$ min.

$105.5 \mathrm{~dB} \quad 108.5 \mathrm{~dB}$ min.

$107 \mathrm{~dB} \quad 110 \mathrm{~dB}$ typ. 


\section{REFERENCES}

1. P.R. Hartmann, J.A. Crosset, "A $90 \mathrm{Mb} / \mathrm{s}$ Digital Transmission System at $11 \mathrm{GHz}$ using 8 PSK Modulation.” Record of ICC, pp. 18.8 - 18.12, Volume II, 1976.

2. P.R. Hartmann, E.W. Allen, "An Adaptive Equalizer for Correction of Multipath Distortion in a $90 \mathrm{Mb} / \mathrm{s} 8$ PSK Microwave System." Record of ICC, pp.5.6, Volume I, 1979.

3. B.C. Ruthroff, "Multiple-Path Fading on Line-of-Sight Microwave Radio Systems as a Function of Path Length and Frequency." Bell System Tehnical Journal, pp. 2375 2398, Volume 50, No. 7, September 1971.

4. M. Ramadam, "Availability Prediction of 8 PSK Digital Microwave System During Multipath Propagation.” Record of ICC, P. 32.4, Volume II, 1979.

5. T.S. Giuffrida, "Measurements of the Effects of Propagation on Digital Radio System Equipped with Space Diversity and Adaptive Equalization." Record of ICC, p 48.1, Volume 3, 1979.

6. C.W. Lundgreen, W.D. Rummler, "Digital Radio Outage due to Selective Fading Observation vs. Prediction from Laboratory Simulation." Bell System Technical Journal, pp. 1073 - 1100, Volume 58, No. 5, May-June 1979. 